Supporting Information

\title{
Pseudocapacitive Properties of Two-Dimensional Surface Vanadia Phases Formed Spontaneously on Titania
}

\author{
Mojtaba Samiee and Jian Luo* \\ Department of NanoEngineering; Program of Materials Science and Engineering \\ University of California, San Diego \\ La Jolla, CA 92093-0448, USA
}

*Email: jluo@alum.mit.edu 


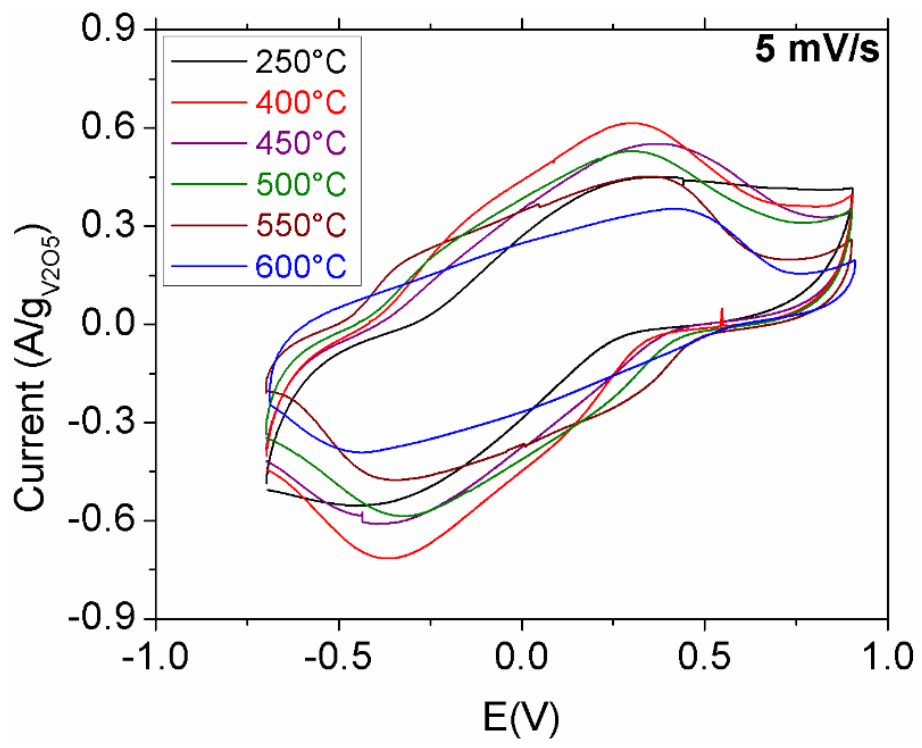

Figure S1. Cyclic voltammograms of the specimens annealed at different temperatures (as labeled in the graph), swept at a scan rate of $5 \mathrm{mV} / \mathrm{s}$. 


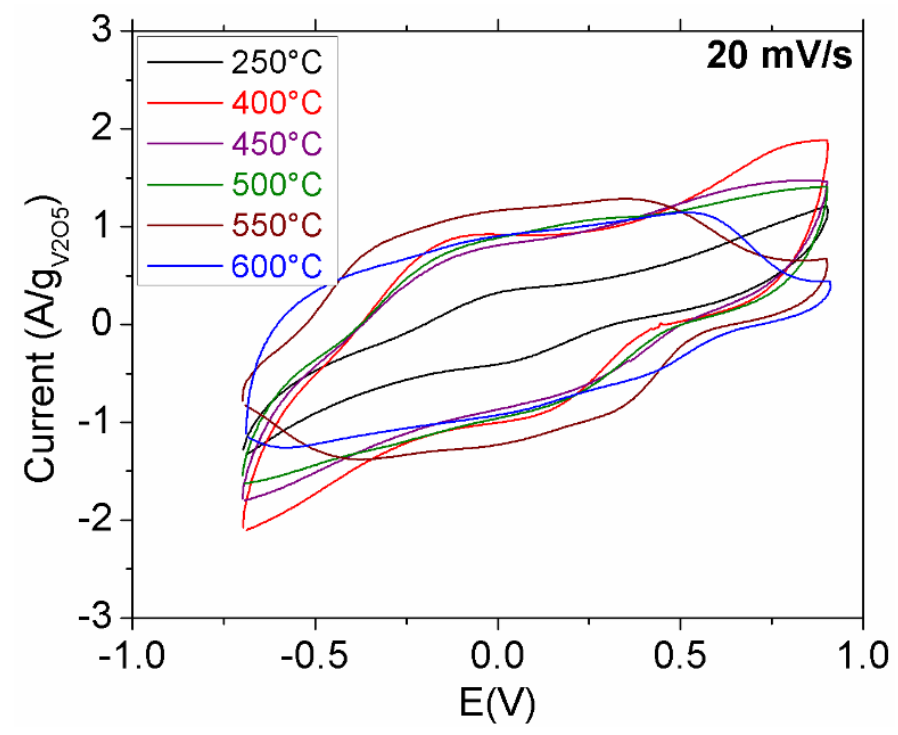

Figure S2. Cyclic voltammograms of the specimens annealed at different temperatures (as labeled in the graph), swept at a scan rate of $20 \mathrm{mV} / \mathrm{s}$. 


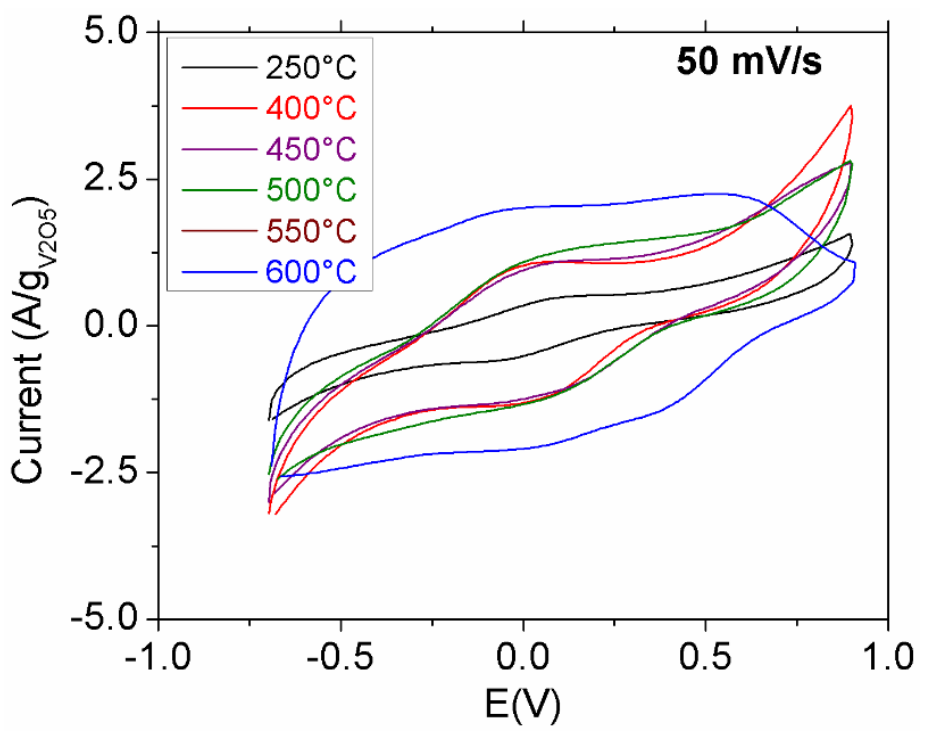

Figure S3. Cyclic voltammograms of the specimens annealed at different temperatures (as labeled in the graph), swept at a scan rate of $50 \mathrm{mV} / \mathrm{s}$. 


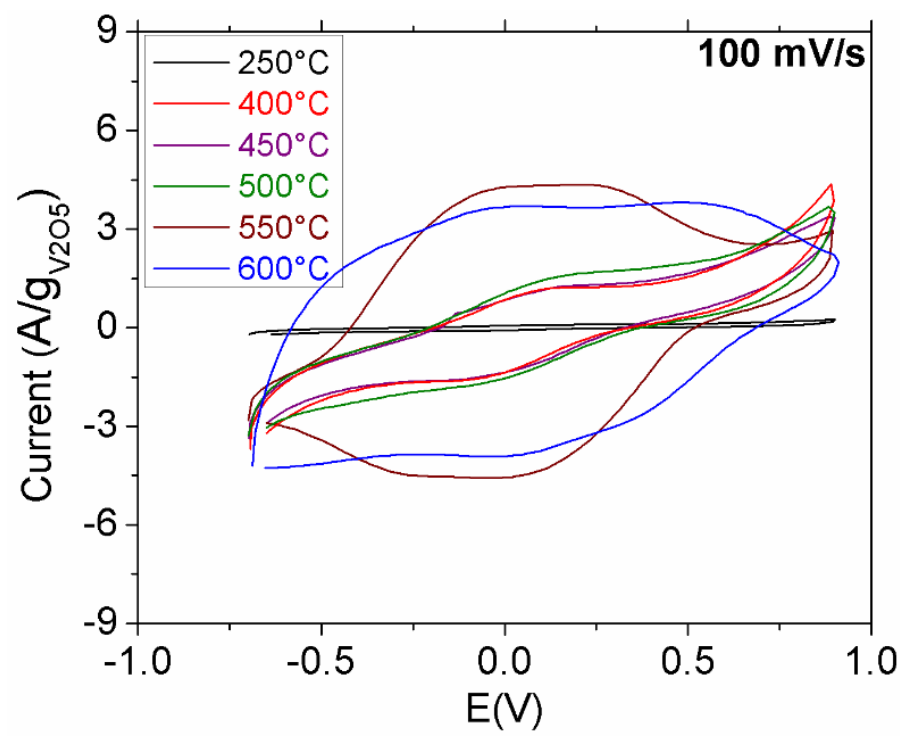

Figure S4. Cyclic voltammograms of the specimens annealed at different temperatures (as labeled in the graph), swept at a scan rate of $100 \mathrm{mV} / \mathrm{s}$. 


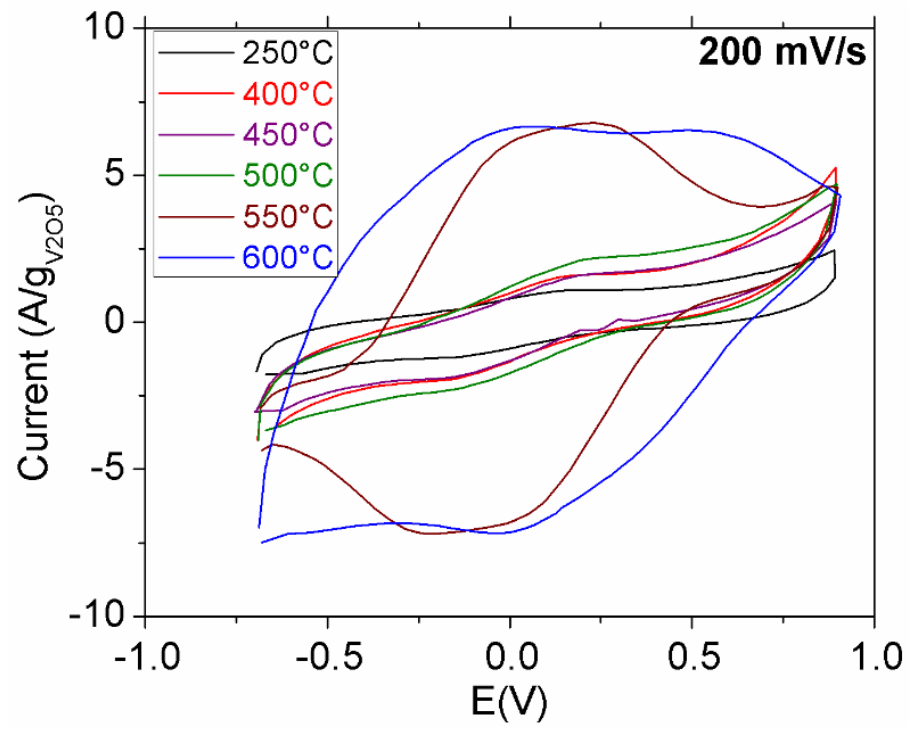

Figure S5. Cyclic voltammograms of the specimens annealed at different temperatures (as labeled in the graph), swept at a scan rate of $200 \mathrm{mV} / \mathrm{s}$. 


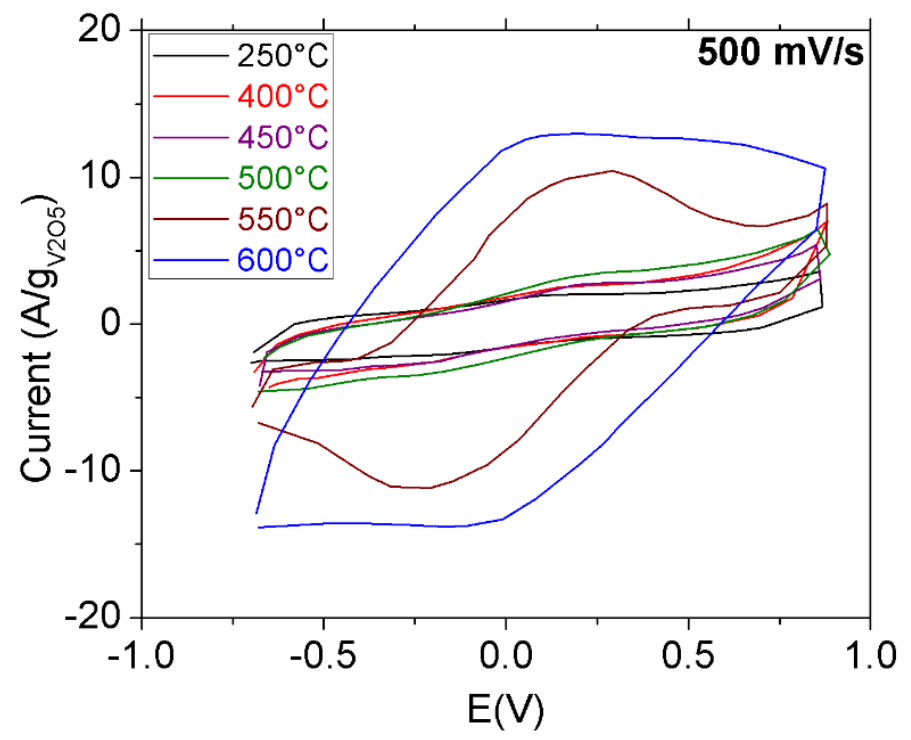

Figure S6. Cyclic voltammograms of the specimens annealed at different temperatures (as labeled in the graph), swept at a scan rate of $500 \mathrm{mV} / \mathrm{s}$. 


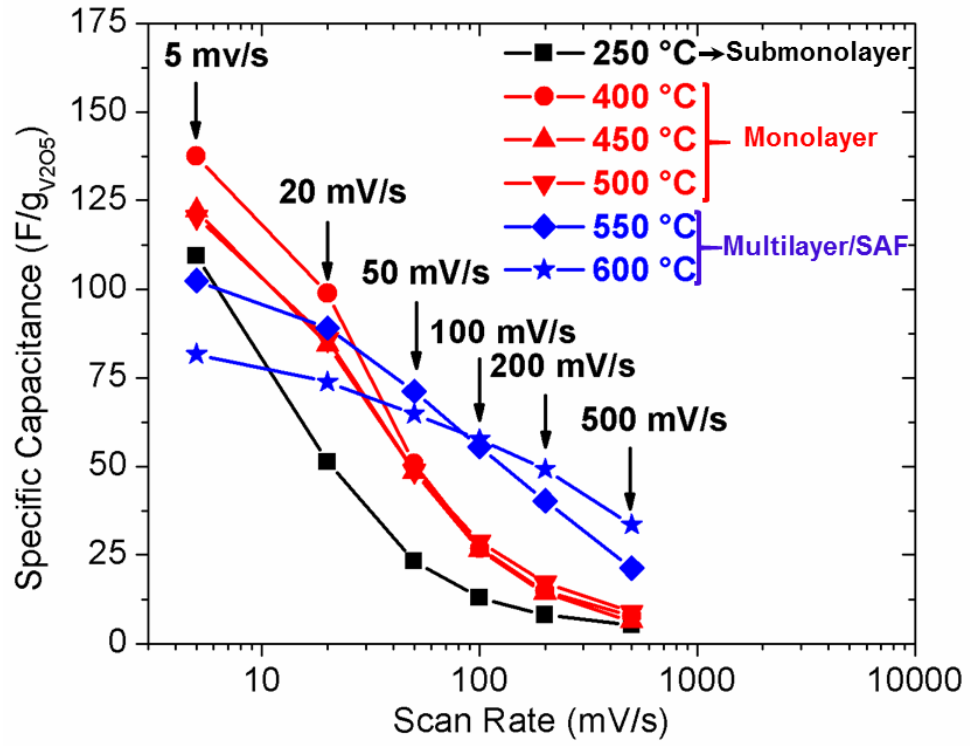

Figure S7. The measured specific capacitances vs. different scan rates for six different specimens (annealed at different temperatures labeled in the graph) in $0.1 \mathrm{M} \mathrm{K}_{2} \mathrm{SO}_{4}$ electrolyte. 